\title{
Ursolic Acid and Luteolin-7-Glucoside Improve Lipid Profiles and Increase Liver Glycogen Content through Glycogen Synthase Kinase-3
}

\author{
Marisa F. Azevedo ${ }^{1}$, Çagri Camsari ${ }^{1, a}$, Carla M. Sá ${ }^{1}$, Cristovao F. Lima ${ }^{2}$, \\ Manuel Fernandes-Ferreira ${ }^{2}$ and Cristina Pereira-Wilson ${ }^{1}$,* \\ ${ }^{1}$ CBMA - Centre of Molecular and Environmental Biology/Department of Biology, University of Minho, Campus de Gualtar, \\ Braga, Portugal \\ ${ }^{2}$ CITAB - Centre for the Research and Technology of Agro-Environmental and Biological Sciences/Department of Biology, \\ University of Minho, Braga, Portugal
}

\begin{abstract}
In the present study, two phytochemicals - ursolic acid (UA) and luteolin-7-glucoside (L7G) - were assessed in vivo in healthy rats regarding effects on plasma glucose and lipid profile (total cholesterol, HDL and LDL), as well as liver glycogen content, in view of their importance in the aetiology of diabetes and associated complications. Both UA and L7G significantly decreased plasma glucose concentration. UA also significantly increased liver glycogen levels accompanied by phosphorylation of glycogen synthase kinase-3 (GSK3). The increase in glycogen deposition induced by UA (mediated by GSK3) could have contributed to the lower plasma glucose levels observed. Both compounds significantly lowered total plasma cholesterol and low-density lipoprotein levels, and, in addition, UA increased plasma high-density lipoprotein levels. Our results show that UA particularly may be useful in preventable strategies for people at risk of developing diabetes and associated cardiovascular complications by improving plasma glucose levels and lipid profile, as well as by promoting liver glycogen deposition. Copyright $@ 2010$ John Wiley \& Sons, Ltd.
\end{abstract}

Keywords: Lamiaceae species; ursolic acid; luteolin-7-glucoside; lipid profile; liver glycogen; functional foods.

\section{INTRODUCTION}

Glucose is a major source of energy for humans and its concentration in the blood is tightly regulated by the pancreatic hormone, insulin. Diabetes mellitus is a metabolic disorder characterized by chronically elevated blood glucose associated with the impairment of insulin secretion and/or a deficient action on peripheral tissues (Klover and Mooney, 2004). Once absorbed after a meal, rising plasma glucose stimulates insulin release and, in tissues such as liver and skeletal muscle, glucose uptake and glycogen synthesis is promoted, which constitutes an important mechanism of glycaemic control. In diabetic patients, this mechanism of plasma glucose clearance is less efficient, originating hyperglycaemia (Postic et al., 2004).

In addition to hyperglycaemia, diabetic patients often show an abnormal plasma lipid profile with high levels of low-density lipoprotein (LDL) cholesterol and lower than desirable levels of high-density lipoprotein (HDL) cholesterol, a combination implicated in the development of cardiovascular complications (Kastelein, 2005;

\footnotetext{
* Correspondence to: Cristina Pereira-Wilson, CBMA- Centre of Molecular and Environmental Biology/Department of Biology, University of Minho, Campus de Gualtar, 4710-057 Braga, Portugal.

E-mail: cpereira@bio.uminho.pt

${ }^{\text {a }}$ Present address: Department of Physiology, Faculty of Veterinary Medicine, University of Ankara, 06110 Ankara, Turkey.
}

Mooradian, 2009). Although not totally elucidated, insulin resistance has been implicated in the pathogenesis of diabetic dyslipidemia (Mooradian, 2009). By reducing insulin resistance as well as by lowering plasma glucose and LDL cholesterol on the one hand, and increasing HDL cholesterol levels on the other, an improvement in the diabetic health status may be achieved that would also help prevent cardiovascular complications. The fact that the number of cases of diabetes is increasing worldwide and the realization that the therapeutic drugs currently in use are not $100 \%$ efficient, motivates the search for new active principles among natural compounds from medicinal plants. In addition, Type 2 diabetes mellitus (T2DM) has been considered a preventable disease through lifestyle changes that includes diet interventions (Costacou and Mayer-Davis, 2003). Natural compounds may be well accepted and should be considered for the production of enriched added-value functional foods to include in dietary strategies for prevention of T2DM and/or health improvement of diabetic patients.

Based on ethnopharmacological data and pharmacological studies, the genus Salvia (Lamiaceae family) has been suggested to possess antidiabetic properties (Baricevic and Bartol, 2000). Previous studies have demonstrated Salvia officinalis tea to decrease fasting blood glucose levels in healthy animals (Lima et al., 2006a). Moreover, methanolic and ethanolic extracts of $S$. officinalis as well as an infusion of $S$. fruticosa have been shown to have hypoglycaemic effects in diabetic 


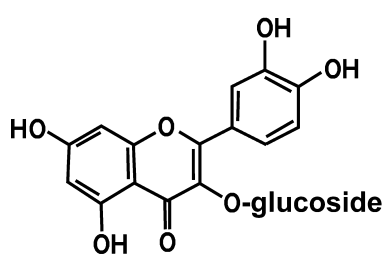

Luteolin-7-O-glucoside

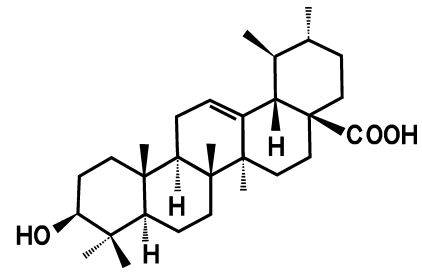

Ursolic acid
Figure 1. Chemical structures of the compounds used in this study: the flavonoid luteolin-7-glucoside and the pentacyclic triterpenoid ursolic acid.

experimental animals (Perfumi et al., 1991; AlarconAguilar et al., 2002; Eidi et al., 2005). Luteolin-7-glucoside (L7G) and ursolic acid (UA) (Fig. 1) are a flavonoid and a triterpenoid, respectively, present in many plants and particularly abundant in Salvia species (Lima et al., 2005; Janicsak et al., 2006), and may contribute to these plants' biological effects (Baricevic and Bartol, 2000).

Many effects attributed to natural compounds, in particular to flavonoids, result from in vitro studies, which may not be observed in vivo due to biotransformation reactions and low bioavailability. Therefore, it is of great interest to assess possible beneficial effects of isolated natural compounds in in vivo studies.

In the present study, the effects of L7G and UA were monitored in vivo in healthy rats regarding plasma glucose, plasma lipid profile (total cholesterol, HDL and LDL) and liver glycogen content in view of their importance in the aetiology of diabetes and associated complications. The expression of glycogen synthase kinase-3 (GSK3) in the liver was also measured since it is an important enzyme involved in the insulin sensitive glycogen synthesis pathway (Lee and Kim, 2007).

\section{MATERIAL AND METHODS}

Chemicals. Bradford Reagent and ursolic acid were purchased from Sigma-Aldrich (St Louis, MO, USA). Luteolin-7-O-glucoside was purchased from Extrasynthese (Genay, France). Glucofix was purchased from A. Menarini Diagnostics (Firenze, Italy). Commercial kits to measure total cholesterol, LDL cholesterol and HDL cholesterol were acquired from Spinreact (Girona, Spain). All others reagents were of analytical grade.

Animals. Male Wistar rats (6 weeks old) were purchased from Charles River Laboratories (Barcelona, Spain) and acclimated to our laboratory animal facilities for at least one week before the start of the experiment. During the experimental period, animals were maintained on a natural light/dark cycle at $20 \pm 2^{\circ} \mathrm{C}$ and given food and tap water ad libitum. Animals were kept and handled in accordance with our university regulations that follow NIH guidelines (NIH Publication No. 80-23; revised 1978) for the experimental use and care of laboratory animals.

Experimental design. Fifteen male Wistar rats were divided into three groups: control; UA-supplemented diet and L7G-supplemented diet. Test compounds were administered orally, once a day, for 7 consecutive days, well mixed in a small piece of food (control group received vehicle only) in a daily dose of $2 \mathrm{mg}$ of compound per $\mathrm{kg}$ of animal body weight. The dose of the compound administered was based on estimations of physiological concentrations by Hertog et al. (1993). During the experiment, water was given ad libitum to the animals. The administration of each compound did not change food and beverage consumption, as well as animal body weights when compared to the control. At the end of the treatment, animals were sacrificed by decapitation. Blood samples were collected and plasma kept for the measurement of glucose and lipid parameters (total cholesterol, LDL cholesterol, HDL cholesterol). Liver samples were also collected, frozen in liquid nitrogen and kept at $-80^{\circ} \mathrm{C}$ for further analyses.

Quantification of plasma glucose, total cholesterol, lowdensity lipoprotein and high-density lipoprotein cholesterol levels. The amount of glucose in rat plasma was measured using a colorimetric enzymatic method - Glucofix - following manufacturer's specifications.

The plasma total cholesterol, LDL cholesterol and HDL cholesterol levels were measured in rat plasma using spectrophotometric commercial kits from Spinreact following manufacturer's specifications.

Liver glycogen quantification. The liver glycogen content was quantified by the amyloglucosidase method as described by Keppler and Decker (1974). Dilutions of the liver homogenate were used to ensure that the determination was done within the linear phase. Liver glycogen content is expressed in $\mu \mathrm{mol}$ glucose per $\mathrm{g}$ of liver.

Liver homogenates and western blot analysis. A piece of liver was homogenized in cold lysis buffer $(0.5 \%$ NP-40 in $50 \mathrm{mM} \mathrm{Na} \mathrm{HPO}_{4}, \mathrm{pH} 7.4,150 \mathrm{mM} \mathrm{NaCl}$, $2 \mathrm{mM}$ EDTA,) containing protease (1 mM phenylmethylsulfonyl fluoride, $10 \mu \mathrm{g} / \mathrm{ml}$ aprotinin) and phosphatase (20 mM NaF, $20 \mathrm{mM}$ orthovanadate) inhibitors added just before use. The homogenate was then centrifuged at $10,000 \times g$ at $4^{\circ} \mathrm{C}$ for $10 \mathrm{~min}$ and the supernatant collected. The amount of protein was measured using Bradford Reagent following manufacturer instructions, using BSA as a standard.

For western blot, $25 \mu \mathrm{g}$ of protein of each sample were separated by SDS-PAGE, transferred onto Hybond-P polyvinylidene difluoride membranes (GE Healthcare, Buckinghamshire, UK) and then blocked in $5 \%(\mathrm{w} / \mathrm{v})$ non-fat dry milk in TPBS $(0.05 \%(\mathrm{v} / \mathrm{v})$ Tween 20 in PBS). The membranes were then probed using a rabbit polyclonal antibody to rat phosphoGSK3 $\alpha / \beta$ (Ser21/9) (Cell Signalling Technology, Inc., Danvers, MA, USA), mouse monoclonal antibody to rat total GSK3 $\alpha / \beta$ (Santa Cruz Biotechnology, Inc., Santa Cruz, CA, USA), and mouse monoclonal anti- $\beta$ actin (Sigma-Aldrich Inc., St Louis, MO, USA). After incubation with the secondary antibody, immunoreactive bands were developed using a chemiluminescence detection system, the Chemi Doc XRS (BioRad Laboratories, Inc., Hercules, CA, USA). Band area intensity was quantified using the Quantity One software from BioRad.

Statistical analysis. Results are expressed as the mean \pm standard error of the mean (SEM) for the number 
A

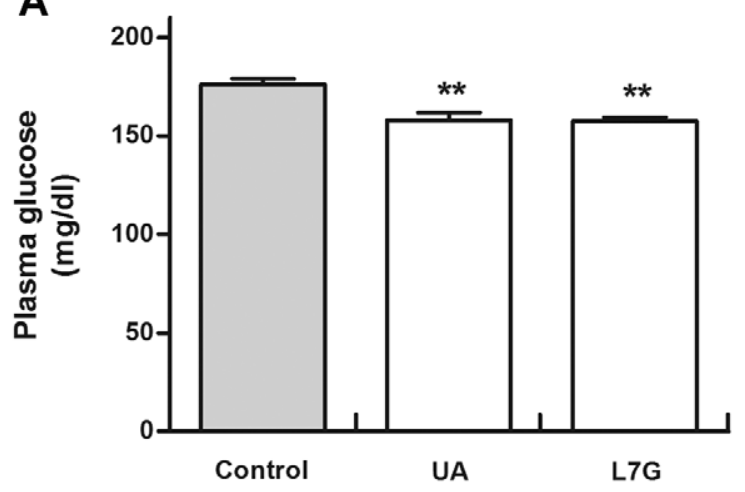

B

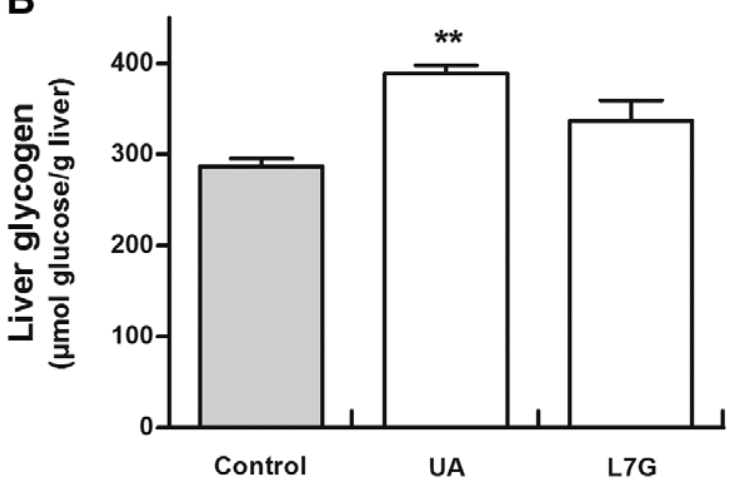

Figure 2. Effect of 7 days of diet supplied with the test compound (UA or L7G) on rat plasma glucose concentration (A), and liver glycogen content (B). Values are expressed as mean \pm SEM $(n=5)$. ${ }^{*}, \mathrm{P}<0.01$ when compared with the control group.

of animals in the group. Statistical significances between groups were determined using the one-way ANOVA followed by the Newman-Keuls multiple comparison test. $P$ value $<0.05$ was considered statistically significant.

\section{RESULTS AND DISCUSSION}

In the present study, we aimed to identify active principles among the constituents of plants to be used in dietary approaches that offer low-cost alternatives in interventions to prevent diabetes or limit its progression. For that, two individual constituents (UA and L7G) present in Salvia species - plants to which antidiabetic properties are attributed - were used at relatively low concentrations (to mimic the normal intake in the human diet) in order to evaluate in vivo effects on plasma glucose, lipid profile and liver glycogen in healthy rats.

After 7 days' treatment (with $2 \mathrm{mg} \cdot \mathrm{kg}^{-1} \cdot \mathrm{day}^{-1}$ of UA or L7G), plasma glucose levels were significantly decreased from $176.1 \mathrm{mg} / \mathrm{dl}(9.78 \mathrm{mM})$ in control group to $158.1 \mathrm{mg} / \mathrm{dl}(8.78 \mathrm{mM})$ in UA and $157.6 \mathrm{mg} / \mathrm{dl}$ (8.76 mM) in L7G (Fig. 2A). A previous study reported that UA prevents glucose intolerance in high-fat-fed mice (Jayaprakasam et al., 2006), corroborating our data that this triterpenoid may interfere with glucose clearance mechanisms. UA and L7G also increased the glycogen content in liver, which was significant for UA (Fig. 2B). Therefore, the increase in glycogen deposition induced mainly by UA could have contributed to the lower plasma glucose levels observed. Recently, in vitro studies performed by Jung et al. (2007) in cultured adipocytes and $\mathrm{CHO} / \mathrm{IR}$ cells showed that UA is an insulin sensitizer, leading to an increase in insulin receptor (IR) $\beta$ autophosphorylation and a subsequent activation of downstream phosphatidylinositol 3-kinase (PI3K) signaling pathway. The activation of this pathway would result in phosphorylation and inactivation of GSK3, leading to an increase glycogen synthase activation and glycogen synthesis (Lee and Kim, 2007). In agreement with this, the increase of liver glycogen induced by UA observed in the present study was accompanied by an increase of liver phospho-GSK3 expression (Fig. 3), indicating that in vivo this compound may also be activating IR and the PI3K signaling.
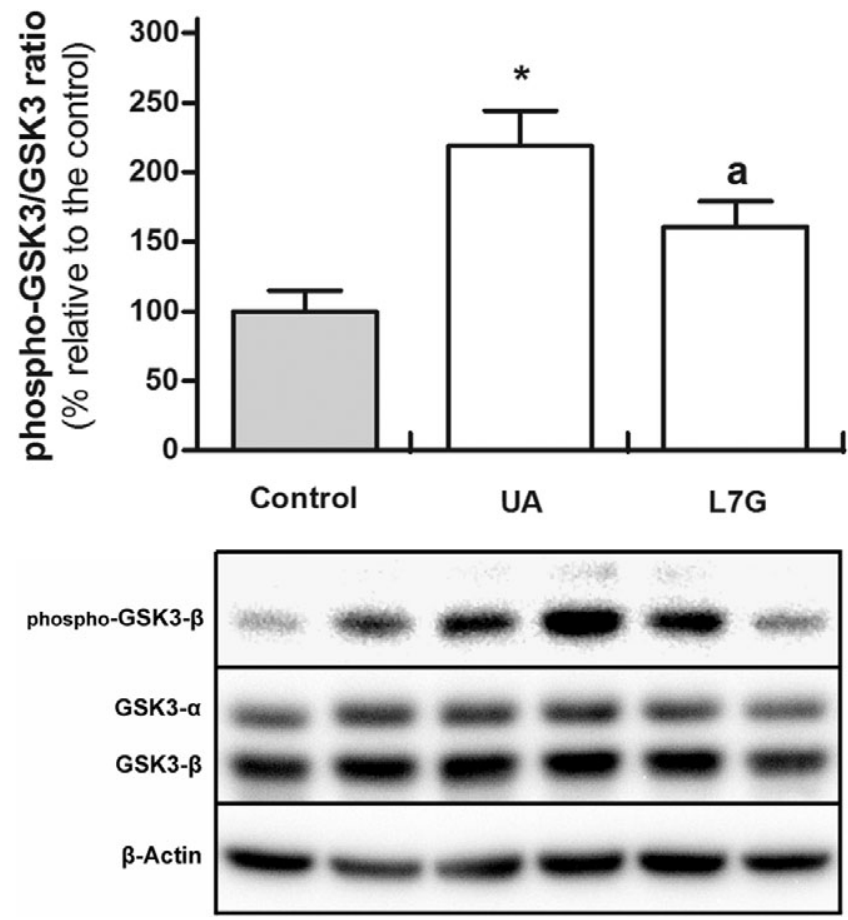

Figure 3. Western blot analysis of phospho-GSK3- $\beta$ protein in liver homogenates of rats treated by 7 days of diet supplied with the test compound UA or L7G $(A)$, with representative immunoblots and corresponding loading control ( $\beta$-actin) from a pair of animals from each treatment $(B)$. Values are expressed as mean \pm SEM $(n=5) .{ }^{*} P<0.05$ when compared with the control group. ${ }^{a} \mathrm{P}=0.06$ when compared with the control group (in this case analysed by the Student's $t$-test).

It seems, therefore, that UA may be useful in the prevention of T2DM, and probably in its treatment where patients show a decrease in insulin response due to lower IR and insulin receptor substrate-1-phosphorylation with decreased PI3K activity (Goodyear et al., 1995; Pratipanawatr et al., 2001).

Although to a smaller extent, L7G also induced the phosphorylation of GSK3 $(P=0.06$, Fig. 3). Flavonoids have shown different effects with respect to the regulation of insulin signaling pathway, which suggest the effects may depend on the compound's concentration and be cell type and context specific. For example, certain flavonoids such as luteolin (the aglycone of L7G), quercetin, apigenin and kaempferol showed inhibitory effects on insulin signaling pathway in mouse 
A

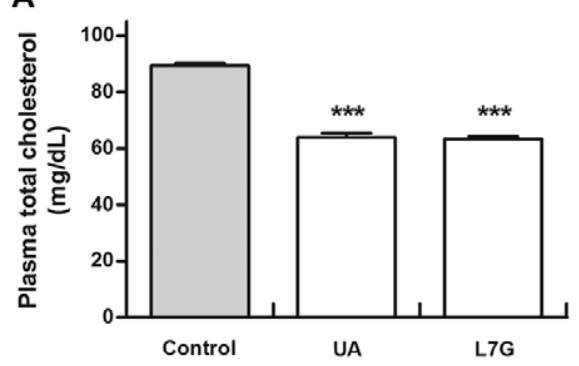

C

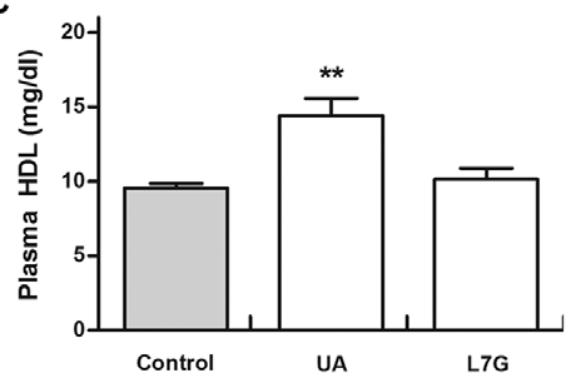

B

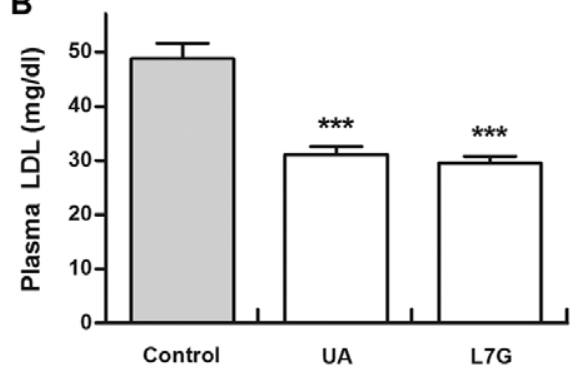

D

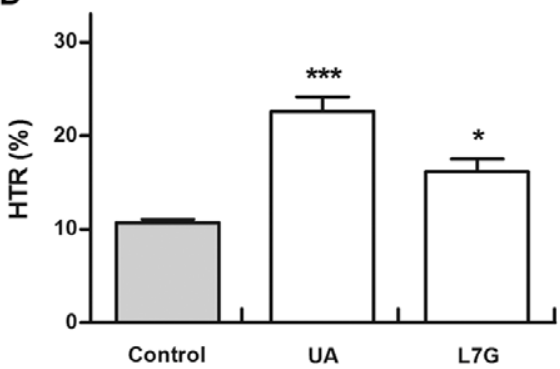

Figure 4. Effects of 7 days of diet supplied with the test compound (UA or L7G) on rat plasma total cholesterol (A), LDL cholesterol (B) and HDL cholesterol (C). HTR $(\%)=(\mathrm{HDL} / T$ otal cholesterol $) \times 100$. Values are expressed as $\mathrm{mean} \pm \mathrm{SEM}(n=5)$. ${ }^{* *}, \mathrm{P}<0.001 ; * *$, $\mathrm{P}<0.01$ and ${ }^{*}, \mathrm{P}<0.05$ when compared with the control group.

adipose cells by suppressing insulin receptor phosphorylation and subsequent inhibition of PI3K and Akt activation (Nomura et al., 2008). Moreover, in colon cancer cells, the flavonoids luteolin and quercetin, as well as the triterpenoid UA, inhibited the PI3K/Akt pathway (Xavier et al., 2009). On the other hand, kaempferol-3neohesperidoside stimulates glycogen synthesis in rat soleus muscle, possibly through PI3K/GSK3 pathway (Cazarolli et al., 2009). As well, epigallocatechin-gallate showed stimulatory effects on insulin pathway inhibiting gluconeogenesis and GSK3 activity in hepatocytes through the activation of PI3K/Akt-dependent pathway (Waltner-Law et al., 2002; Lin and Lin, 2008).

In addition to plasma glucose, plasma lipids are important predictors of T2DM progression and development of cardiovascular complications. In order to assess possible effects of UA and L7G on lipid profile, levels of plasma lipids (total cholesterol, LDL and HDL) were measured. As shown in Fig. 4A, total plasma cholesterol levels were significantly reduced by both treatments (by $28.5 \%$ with UA and by $29.2 \%$ with L7G), which was accompanied by a consistent reduction of plasma LDL levels (by $36.2 \%$ and $39.5 \%$, respectively, Fig. 4B). In addition, a $51.1 \%$ increase of HDL plasma levels was induced by UA treatment while L7G was not active on this parameter (Fig. 4C). Both UA and L7G increased significantly the HDL/total cholesterol ratio (HTR in \%), although effects were more pronounced for UA (Fig. 4D). Thus, both compounds showed relevant effects on the control of plasma lipids, by diminishing total cholesterol and LDL with UA additionally increasing HDL levels. Interestingly, in a pilot study where human volunteers at risk of developing T2DM were treated with $S$. officinalis tea (L7G being the main flavonoid present in the extract) an improvement of lipid profile was also observed, characterized by decreased levels of plasma total cholesterol and LDL as well as increased levels of HDL (Sá et al., 2009). Statins and fibrates are pharmaceutical drugs currently used in the treatment of diabetic dyslipidaemia
(Kastelein, 2005). Since statins act mainly by reducing LDL cholesterol levels whereas fibrates increase HDL cholesterol levels, combination therapies have been used to control dyslipidaemia and to diminish the risk of cardiovascular diseases and associated morbidity and mortality (Kastelein, 2005). UA administered alone produced this double effect in rats, which warrants future studies in diabetic/obese animals and humans.

Thiazolidinediones (TZDs) - also used in the treatment of T2DM patients - act through peroxisome proliferator-activated receptor- $\gamma$ improving insulin sensitivity and lipid profile (Levetan, 2007; Kersten, 2008). In addition, recently Ciaraldi et al. (2006) showed that the TZD troglitazone also inhibited GSK3 in skeletal muscle of obese T2DM patients. In the present study, although probably through different mechanisms, UA was also effective on both the inhibition of GSK3 activity (with a concomitant rise in liver glycogen synthesis) and on the improvement of lipid profile. Since insulin resistance may have a central role in the development of diabetic dyslipidemia (Mooradian, 2009), the possible induction of insulin sensitivity by UA may also be indirectly involved in the improvement of lipid profile.

The effects of UA and L7G, in particular those of UA, suggest, therefore, that these compounds could contribute positively to prevention and also the control of dyslipidaemia and hyperglycaemia observed in T2DM. These beneficial effects of UA and L7G may be assisted by their known effects on the enhancement of cellular antioxidant defences (Lima et al., 2006b; Yin and Chan, 2007; Ramos et al., 2008) known to be overwhelmed by the inherent excess of reactive oxygen species production observed in diabetes.

In conclusion, we observed that L7G showed effects in vivo on plasma glucose and lipid profile, whereas UA in addition to these effects also increased liver glycogen deposition and plasma HDL levels. Considering that T2DM and cardiovascular diseases can be prevented through lifestyle changes including dietary strategies, 
these two phytochemicals may be good candidates for the production of functional foods and food supplements due to their beneficial properties on relevant parameters for the prevention and control of these disorders. In addition, since these compounds are present in many Salvia species, they may account for the health benefits attributed to this genus, in particular for its antidiabetic reputation.

\section{Acknowledgements}

MFA and CMS were supported by the Foundation for Science and Technology, Portugal, through the grants SFRH/BD/12527/2003 and SFRH/BD/42566/2007, respectively. This work was supported by the Foundation for Science and Technology, Portugal, research grant POCI/AGR/62040/2004.

\section{REFERENCES}

Alarcon-Aguilar FJ, Roman-Ramos R, Flores-Saenz JL, AguirreGarcia F. 2002. Investigation on the hypoglycaemic effects of extracts of four Mexican medicinal plants in normal and alloxandiabetic mice. Phytother Res 16: 383-386.

Baricevic D, Bartol T. 2000. The biological/pharmacological activity of the Salvia genus. In SAGE - The Genus Salvia, Kintzios SE (ed.). Harwood Academic Publishers: Amsterdam; 143-184.

Cazarolli LH, Folador P, Pizzolatti MG, Mena Barreto Silva FR. 2009. Signaling pathway of kaempferol-3-neohesperidoside in glycogen synthesis in rat soleus muscle. Biochimie $\mathbf{9 1}$ 843-849.

Ciaraldi TP, Oh DK, Christiansen L, Nikoulina SE, Kong AP, Baxi S, Mudaliar S, Henry RR. 2006. Tissue-specific expression and regulation of GSK-3 in human skeletal muscle and adipose tissue. Am J Physiol Endocrinol Metab 291: E891-E898.

Costacou T, Mayer-Davis EJ. 2003. Nutrition and prevention of type 2 diabetes. Annu Rev Nutr 23: 147-170.

Eidi M, Eidi A, Zamanizadeh H. 2005. Effect of Salvia officinalis L. leaves on serum glucose and insulin in healthy and streptozotocin-induced diabetic rats. J Ethnopharmacol 100: 310-313.

Goodyear LJ, Giorgino F, Sherman LA, Carey J, Smith RJ, Dohm GL. 1995. Insulin receptor phosphorylation, insulin receptor substrate-1 phosphorylation, and phosphatidylinositol 3-kinase activity are decreased in intact skeletal muscle strips from obese subjects. J Clin Invest 95: 2195-2204.

Hertog MG, Hollman PC, Katan MB, Kromhout D. 1993. Intake of potentially anticarcinogenic flavonoids and their determinants in adults in The Netherlands. Nutr Cancer 20: 21-29.

Janicsak G, Veres K, Kakasy AZ, Mathe I. 2006. Study of the oleanolic and ursolic acid contents of some species of the Lamiaceae. Biochem Syst Ecol 34: 392-396.

Jayaprakasam B, Olson LK, Schutzki RE, Tai MH, Nair MG. 2006. Amelioration of obesity and glucose intolerance in highfat-fed C57BL/ 6 mice by anthocyanins and ursolic acid in cornelian cherry (Cornus mas). J Agric Food Chem 54: 243-248.

Jung SH, Ha YJ, Shim EK, Choi SY, Jin JL, Yun-Choi HS, Lee JR. 2007. Insulin-mimetic and insulin-sensitizing activities of a pentacyclic triterpenoid insulin receptor activator. Biochem J 403: 243-250.

Kastelein JJ. 2005. Modifying plasma low-density lipoprotein and high-density lipoprotein cholesterol: what combinations are available in the future? Am $J$ Cardiol 96: $20 \mathrm{~K}-27 \mathrm{~K}$.

Keppler D, Decker K. 1974. Glycogen: determination with amyloglucosidase. In Methods of Enzymatic Analysis, Bergmeyer HU (ed). Weinheim: Verlage Chemie; 1127-1131.

Kersten S. 2008. Peroxisome proliferators activated receptors and lipoprotein metabolism. PPAR Res 2008: 132960.

Klover PJ, Mooney RA. 2004. Hepatocytes: critical for glucose homeostasis. Int J Biochem 36: 753-758.

Lee J, Kim MS. 2007. The role of GSK3 in glucose homeostasis and the development of insulin resistance. Diabetes Res Clin Pract 77: S49-S57.
Levetan C. 2007. Oral antidiabetic agents in type 2 diabetes. Curr Med Res Opin 23: 945-952.

Lima CF, Andrade PB, Seabra RM, Fernandes-Ferreira M, Pereira-Wilson C. 2005. The drinking of a Salvia officinalis infusion improves liver antioxidant status in mice and rats. $J$ Ethnopharmacol 97: 383-389.

Lima CF, Azevedo MF, Araujo R, Fernandes-Ferreira M, PereiraWilson C. 2006a. Metformin-like effect of Salvia officinalis (common sage): is it useful in diabetes prevention? $\mathrm{Br} J$ Nutr 96: 326-333.

Lima CF, Fernandes-Ferreira M, Pereira-Wilson C. 2006b. Phenolic compounds protect HepG2 cells from oxidative damage: relevance of glutathione levels. Life Sci 79: 2056-2068.

Lin CL, Lin JK. 2008. Epigallocatechin gallate (EGCG) attenuates high glucose-induced insulin signalling blockade in human hepG2 hepatoma cells. Mol Nutr Food Res 52: 930939.

Mooradian AD. 2009. Dyslipidemia in type 2 diabetes mellitus. Nat Clin Pract Endocrinol Metab 5:150-159.

Nomura M, Takahashi T, Nagata N, Tsutsumi K, Kobayashi S, Akiba T, Yokogawa K, Moritani S, Myiamoto K. 2008. Inhibitory mechanisms of flavonoide on insulin-stimulated glucose uptake in MC3T3-G2/PA6 adipose cells. Biol Pharm Bull 31: 1403-1409.

Perfumi M, Arnold N, Tacconi R. 1991. Hypoglycemic activity of Salvia fruticosa Mill. from Cyprus. J Ethnopharmacol 34: 135-140.

Pratipanawatr W, Pratipanawatr T, Cusi K, Berria R, Adams JM, Jenkinson CP, Maezono K, DeFronzo RA, Mandarino LJ. 2001. Skeletal muscle insulin resistance in normoglycemic subjects with a strong family history of type 2 diabetes is associated with decreased insulin-stimulated insulin receptor substrate-1 tyrosine phosphorylation. Diabetes 50: 2572-2578.

Postic C, Dentin R, Girard J. 2004. Role of the liver in the control of carbohydrate and lipid homeostasis. Diabetes Metab 30: 398-408.

Ramos AA, Lima CF, Pereira ML, Fernandes-Ferreira M, PereiraWilson C. 2008. Antigenotoxic effects of quercetin, rutin and ursolic acid on HepG2 cells: Evaluation by the comet assay. Toxicol Lett 177: 66-73.

Sá CM, Ramos AA, Azevedo MF, Lima CF, Fernandes-Ferreira M, Pereira-Wilson C. 2009. Sage tea drinking improves lipid profile and antioxidant defences in humans. Int $\mathrm{J} \mathrm{Mol} \mathrm{Sci}$ 10: 3937-3950.

Waltner-Law ME, Wang XL, Law BK, Hall RK, Nawano $M$, Granner DK. 2002. Epigallocatechin gallate, a constituent of green tea, represses hepatic glucose production. $J$ Biol Chem 277: 34933-34940.

Xavier CP, Lima CF, Preto A, Seruca R, Fernandes-Ferreira M, Pereira-Wilson C. 2009. Luteolin, quercetin and ursolic acid are potent inhibitors of proliferation and inducers of apoptosis in both KRAS and BRAF mutated human colorectal cancer cells. Cancer Lett 281: 162-170.

Yin MC, Chan KC. 2007. Nonenzymatic antioxidative and antiglycative effects of oleanolic acid and ursolic acid. J Agric Food Chem 55: 7177-7181. 